\title{
CERTAIN CONGRUENCES ON QUASIGROUPS
}

\author{
H. A. THURSTON
}

1. Using the ideas of $[1],{ }^{1}$ we define a lattice-isomorphism between the reversible congruences on a quasigroup and certain congruences on its group of translations. This may be used to get certain properties of the quasigroup congruences from those of the translation-group congruences; for example, it gives a new proof that reversible congruences on a quasigroup are permutable (a proof of this has been given in [3]).

Notation. A relation $\theta$ in a set $S$ is a set of ordered 2-sets of elements of $S$. If $(a, b) \in \theta$, we say " $a$ is in the relation $\theta$ to $b^{n}$; the shorter notation $a \theta b$ will sometimes be used for this. For example, a mapping $x \rightarrow x \theta$ may be taken to be the set of all $(x, x \theta)$ and is then a relation in this sense.

$\theta^{-1}$ is the set of all $(a, b)$ for which $b \theta a$.

$\theta \phi$ is the set of all $(a, b)$ for which $a \theta c \phi b$ for some $c$.

Clearly $\theta^{-1}$ and $\theta \phi$ are relations in $S$ if $\theta$ and $\phi$ are.

If $q$ is an equivalence (that is, if $q^{-1}=q q=q$ ), then $a q$ is the set of all elements in the relation $q$ to $a$.

2. Given a quasigroup whose set of elements is $S$ it is possible to give definitions ${ }^{2}$ of two operations / and $\backslash$ :

$a / b$ is the $x$ for which $x \cdot b=a$.

$a \backslash b$ is the $x$ for which $a \cdot x=b$.

Clearly

$$
(a / b) \cdot b=a, \quad a \cdot(a \backslash b)=b, \quad(a \cdot b) / b=a, \quad a \backslash(a \cdot b)=b .
$$

On the other hand, if we have an algebra $\varepsilon$ whose set of elements is $S$, whose operations are $\cdot, /$, and $\backslash$, and for which (1) is true, then the algebra $S$ with the operation - and elements $S$ is a quasigroup. $\varepsilon$ is equationally defined: it might possibly be named an equasigroup.

3. Definition. A congruence $q$ on a quasigroup is reversible if (i) $a q b$ whenever $a c q b c$ and (ii) $a q b$ whenever $c a q c b$. Clearly a congruence on $S$ is reversible if and only if it is a congruence on $\varepsilon$. Equally clearly, $S / q$ is a quasigroup under the Kronecker operation - if and only if $q$ is reversible. (The reversible property is needed for cancellation to be possible.)

Received by the editors November 6, 1950 and, in revised form, February 27, 1951.

${ }^{1}$ Numbers in brackets refer to the bibliography at the end of the paper.

${ }^{2}$ The notation is from [2]. 
4. Definitions. $\rho_{a}$ is the mapping $x \rightarrow x \cdot a$, and $\lambda_{a}$ is $x \rightarrow a \cdot x$. The translator, $\Sigma$, of $\mathcal{S}$ (or of $\mathcal{E}$ ) is the group generated by all $\rho_{a}$ and $\lambda_{a}$ for all $a$ of $S$, and is a permutation group on $S$.

5. Now we give a relation between congruences on $\varepsilon$ and congruences on $\Sigma$. Clearly an equivalence $q$ on $S$ is a congruence on $\varepsilon$ if and only if $x \sigma q y \sigma$ whenever $x q y$ and $\sigma \in \Sigma$; that is, if and only if $\sigma^{-1} \mathfrak{q} \subseteq \mathfrak{q}$ for every $\sigma$ of $\Sigma$. From now on the letter $q$ will be used only for congruences on $\varepsilon$.

Definition. $q^{\dagger}$ is the relation in $\Sigma$ for which $\theta q^{\dagger} \phi$ if and only if $\theta^{-1} \phi \subseteq q$.

If $\sigma \in \Sigma$, then $x \mathfrak{q} \rightarrow(x \sigma) \mathfrak{q}$ is a mapping, $\bar{\sigma}$ say, of $S / \mathfrak{q}$ into $S / \mathfrak{q}$. For if $x q=y q$, then $x q y$. Therefore $x \sigma q y \sigma$ and so $x \sigma q=y \sigma q$. The mapping $\sigma \rightarrow \bar{\sigma}$ is a homomorphism (that is, $\sigma \tau \rightarrow \bar{\sigma} \bar{\tau}$ ) and $q^{\dagger}$ is its kernel. Therefore $\mathrm{q}^{\dagger}$ is a congruence on $\Sigma$.

Note. Clearly $q^{\dagger} \supseteq p^{\dagger}$ if $q \supseteq p$.

6. From now on the letter $p$ will be used only for congruences on $\Sigma$.

Definition. $p^{+}$is $\cup \theta^{-1} \phi$ (over all $\theta, \phi$ for which $\theta p \phi$ ).

It is not hard to see that $p^{+}$is a congruence on $\varepsilon$. For (i) clearly $\boldsymbol{p}^{+}=\left(\boldsymbol{p}^{+}\right)^{-1}$. (ii) Let $(a, b) \in\left(\boldsymbol{p}^{+}\right)^{2}$. Then, for some $c, a \boldsymbol{p}^{+} c \boldsymbol{p}^{+} b$. Therefore $a \theta^{-1} \phi c$ and $a \psi^{-1} \chi b$, where $\theta p \phi$ and $\psi p \chi$. Then $a \theta^{-1} \phi=c=b \chi^{-1} \psi$ and so $(a, b) \in \theta^{-1} \phi \psi^{-1} \chi=\left(\phi^{-1} \theta\right)^{-1} \psi^{-1} \chi$. But $\phi^{-1} \theta p \phi^{-1} \phi=\iota=\psi^{-1} \psi p \psi^{-1} \chi$. Therefore $a p^{+} b$, and so $\left(p^{+}\right)^{2} \subseteq p^{\dagger}$.

(iii) Let $(a, b) \in \sigma^{-1} p^{+} \sigma$ where $\sigma \in \Sigma$. Then

$$
\begin{aligned}
(a, b) & \in \sigma^{-1} \theta^{-1} \phi \sigma \\
& =(\theta \sigma)^{-1}(\phi \sigma) \\
& \subseteq p^{+} .
\end{aligned}
$$

(where $\theta p \phi$ )

(where $(\theta \sigma) \boldsymbol{p}(\phi \sigma)$ )

Note. Clearly $\boldsymbol{p}^{\dagger} \supseteq \boldsymbol{q}^{+}$if $\boldsymbol{p} \supset \boldsymbol{q}$.

7. $p \subseteq q^{\dagger}$ if and only if $p^{+} \subseteq q$. For, by the definition of $q^{\dagger}$, $p \subseteq q^{\dagger}$ if and only if (i) $\theta^{-1} \phi \subseteq q$ whenever $\theta p \phi$. And (i) is true, by the definition of $p^{+}$, if and only if $p^{+} \subseteq q$. Then if $p=q^{\dagger}$ we have $p^{+} \subseteq q$, that is $\mathrm{q}^{\dagger+} \subseteq q$. On the other hand, if $a \mathrm{q} b$, let $u$ be any element of $S$ and put $a=u \lambda_{v}, b=u \lambda_{w}$. Then $v q w$ (because $q$ is reversible), and so, for any $x$ of $S, x \lambda_{v} q x \lambda_{w}$. Therefore $\lambda_{v}^{-1} \lambda_{w} \subseteq q$, and so $\lambda_{v} q^{\dagger} \lambda_{w}$. But $(a, b)$ $=\left(u \lambda_{v}, u \lambda_{w}\right) \in \lambda_{0}^{-1} \lambda_{w}$. Therefore $a q^{\dagger+} b$. Therefore $q^{\dagger+} \supseteq q$ and so $q=q^{\dagger+}$. Therefore $\dagger$ is a one-to-one mapping of the set of all congruences on $\varepsilon$ into the set of congruences on $\Sigma$, and $t$ is $(\dagger)^{-1}$. By notes 5 and 6 , this mapping is an isomorphism between the lattice of congruences on $\varepsilon$ and a sublattice of the lattice of congruences on $\Sigma$.

8. Any two congruences on $\mathcal{E}$ are permutable. Let $\mathfrak{p}$ and $\mathfrak{r}$ be any 
two congruences on $\varepsilon$. Any congruence on a group is given by a normal subgroup: let the congruences $\mathfrak{p}^{\dagger}$ and $\mathfrak{r}^{\dagger}$ be given by subgroups $\Pi$ and $\mathrm{P}$. Then, for every $a$ of $S, a \mathfrak{p}=a \Pi$. For if $b \in a \mathfrak{p}$, let $u, v$, and $w$ be as in $\S 7$. Then $b=a \lambda_{0}^{-1} \lambda_{w}$ where $\lambda_{v}^{-1} \lambda_{w} \in \Pi$. Therefore $a p \subseteq a \Pi$. On the other hand, if $b \in a \Pi$, then $b=a \theta$ where $\theta \in \Pi$ and so $\theta p^{\dagger} \iota$. Then $a \theta p a \iota$; that is, $b p a$, and so $b \in a p$. Therefore $a \Pi \subseteq a p$, and so $a \Pi=a \mathfrak{p}$. In the same way, $a \mathrm{P}=a \mathrm{r}$.

Now, if $a \mathrm{pr} b$, then for some $c, a \in q c \mathfrak{p}=c \Pi$ and $c \in b \mathrm{r}=b \mathrm{P}$. Therefore $a \in b \mathrm{P} \Pi=b \Pi \mathrm{P}$. We may now let $a=b \theta \phi$ where $\theta \in \Pi$ and $\phi \in \mathrm{P}$. Then $a \mathfrak{r} b \theta$. But $b \mathfrak{p} b \theta$. Therefore $a \mathfrak{r} p b$. Therefore $\mathfrak{p r} \subseteq \mathfrak{r p} ;$ that is, $\mathfrak{p}$ and $\mathfrak{r}$ are permutable.

9. An important point about this is that proofs have been given (for example, in [4, pp. 87-89]) of the Schreier-Zassenhaus theorem for algebras all of whose congruences are permutable and which have a one-element subalgebra. An equasigroup has not, in general, a oneelement subalgebra, but the theorem is true in this form:

If $E, A_{1}, \cdots, A_{m}$ and $E, B_{1}, \cdots, B_{n}$ are normal series of an equasigroup $E$, and if $A_{m} \cap B_{n} \neq \varnothing$, then the series have isomorphic refinements.

\section{BIBLIOGRAPHY}

1. A. A. Albert, Quasigroups. I, Trans. Amer. Math. Soc. vol. 54 (1943) p. $507 \cdot$

2. T. Evans, Homomorphisms of non-associative systems, J. London Math. Soc' vol. 24 (1949) p. 254.

3. G. Trevisan, $A$ proposito delle relazioni di congruenza sui quasi-gruppi, Rendiconti del Seminario Matematico della Università di Padova vol. 19 (1950) pp. 367-370.

4. G. Birkhoff, Lattice theory, Amer. Math. Soc. Colloquium Publications, vol. 25, rev. ed., 1948.

UNIVERSITY OF BRISTOL 